\title{
Osteoporosi nei pazienti con diabete mellito
}

\section{Osteoporosis and diabetes mellitus}

\section{Andrea Montagnani *, Massimo Alessandri}

Ambulatorio delle Malattie Metaboliche Ossee e Osteoporosi, UO Medicina Interna, Ospedale della Misericordia, AUSL 9, Grosseto

Ricevuto il 21 febbraio 2010; accettato il 16 luglio 2010

disponibile online il 19 novembre 2010

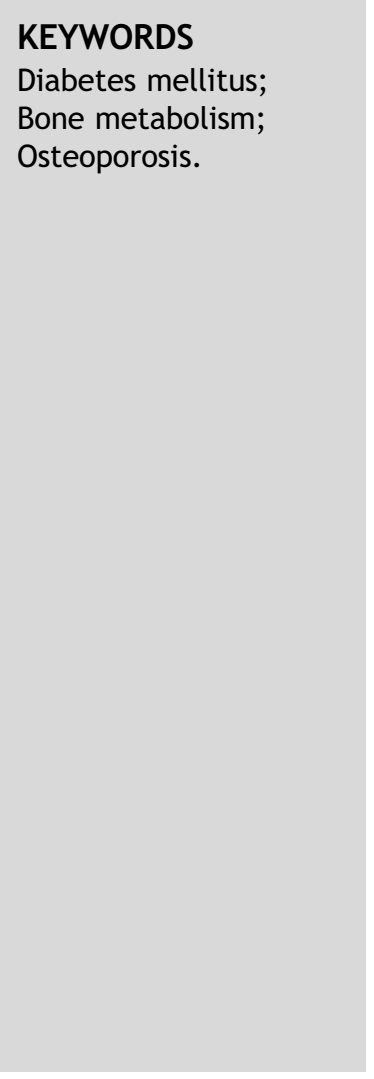

\begin{abstract}
Summary
Introduction: Diabetes mellitus (DM) and osteoporotic fractures are major causes of mortality and morbidity in older subjects. Recent reports have revealed close association between fracture risk and DM types 1 and 2 (DM1 and DM2, respectively). Aim of this review is to highlight the importance of these diseases in the elderly and examine certain etiopathogenetic aspects of DMassociated osteoporosis, which could be useful in management of diabetic patients.

Materials and methods: We searched the Embase and PubMed databases using diabetes, osteoporosis, and bone mineral density (BMD) as search terms and 1989-2009 as publication dates.

Discussion: The risk of fractures seems to be increased in both types of DM although DM2 seems to be associated with normal-high BMDs compared with the normal population.

This apparent paradox could reflect greater bone frailty in diabetic patients that are unrelated to adipose tissue, hyperinsulinemia, deposition of advanced glycosylation end products in collagen, reduced serum IGF-1 levels, hypercalciuria, renal failure, microangiopathy, and/or inflammation. Diabetic patients' propensity to fall and multiple comorbidities might also explain their higher fracture rates. The effects of drugs that inhibit bone resorption in diabetic patients are probably similar to those obtained in nondiabetics although there is little information on this issue. In general, effective treatment of diabetes has positive effects on bone metabolism. Metformin acts directly on bone tissue, reducing AGE accumulation, and insulin has direct effects on osteoclast activity. In contrast, the thiazolidinediones seem to have negative effects since they orient mesenchymal progenitor cell differentiation toward adipose rather than bone tissue. Incretin therapy is a newer approach that appears to modify interactions between nutrition and bone turnover (e.g., postprandial suppression of bone resorption).

Conclusions: Better understanding of how diabetes and its treatment influence bone tissue could lead to more effective strategies for preventing fractures in diabetic patients. More investigation is needed to determine whether conventional osteoporotic therapy is fully effective in patients with DM.

(c) 2010 Elsevier Srl. All rights reserved.
\end{abstract}

* Corrispondenza: Medicina Interna, Ospedale della Misericordia, via Senese - 58100 Grosseto.

E-mail: montagnaniand@gmail.com (A. Montagnani). 


\section{Cenni epidemiologici}

L'osteoporosi e il diabete mellito sono due patologie frequenti, con eziologia multifattoriale e a maggiore prevalenza nella popolazione anziana. Le fratture osteoporotiche sono associate a un significativo aumento della mortalità, della morbilità e a una conseguente riduzione della qualità di vita $[1,2]$. I principali fattori di rischio notoriamente associati alle fratture osteoporotiche sono l'età avanzata, il basso indice di massa corporea e l'anamnesi familiare positiva, le fratture prevalenti, la terapia steroidea, il fumo di sigaretta [3]. Al contrario, malgrado numerosi studi di tipo osservazionale abbiano approfondito il rapporto tra diabete e rischio di frattura, il ruolo del diabete come fattore di rischio per osteoporosi e frattura da fragilità rimane a oggi non del tutto chiarito [4].

La densità minerale ossea (BMD) è un forte predittore del rischio di frattura, sebbene non sia l'unica componente a determinare la resistenza fisica del tessuto osseo. Il diabete mellito di tipo 1 sembra associato a una ridotta BMD, ma anche a un'alterata qualità ossea, aspetto non valutabile con la sola densitometria a raggi $\times(D X A)$. La BMD risulta ridotta nei pazienti affetti da diabete di tipo 1 (DM1) nella maggior parte degli studi [5-7], ma non in tutti $[8,9]$. A livello dello scheletro assiale, il DM1 risulta associato a una riduzione della BMD in sede sia lombare sia femorale [7,10-12]. La perdita della massa ossea avviene prevalentemente durante $i$ primi anni di malattia, cosicché i pazienti che sviluppano il diabete in età pediatrica non raggiungono un picco di massa ossea adeguato con un conseguente incremento del rischio di osteoporosi nell'età adulta [12].

Per quanto riguarda la relazione tra diabete di tipo 2 (DM2) e BMD, esistono dati contrastanti [6,7,13-21]; la maggioranza degli studi riporta una BMD normale o leggermente aumentata nei pazienti diabetici rispetto alla popolazione sana di controllo. Questo dato risulta più evidente nelle donne che negli uomini [20] e si mantiene anche dopo correzione per fattori confondenti come il peso corporeo.

Sebbene siano numerosi gli studi che hanno indagato l'associazione tra DM2 e rischio di frattura, non sembra possibile trarre conclusioni definitive. Infatti, alcuni autori hanno riportano un incremento del rischio di frattura nei pazienti diabetici di tipo 2 [18,22-27], mentre altri studi non hanno riscontrato alcuna associazione $[7,14,15]$ e altri ancora hanno riscontrato una relazione inversa $[18,22,23,28]$.

Il rischio di frattura di femore nel DM2 si attesta fra il $18 \%$ negli uomini e l'11\% nelle donne anche dopo correzione per fattori confondenti quali età, comorbilità e farmaci noti per influenzare negativamente il rischio di cadute [29]. Una recente ed estesa metanalisi [4] per un totale di 800.000 soggetti ha confermato questo dato, riportando una correlazione positiva fra diabete mellito e fratture non vertebrali (RR 1,2; IC 95\% 1,01-1,5), fratture femorali (RR 1,7; IC 95\% 1,3-2,2) e fratture del piede (RR 1,3; IC 95\% 1,1-1,7). Tali associazioni rimanevano significative anche dopo aggiustamento per età, attività fisica e indice di massa corporea (RR 2,6; IC 95\% 1,5-4,5), tendevano a essere più forti nell'uomo, anziché nella donna, e nei pazienti con una storia più lunga di malattia. Nello stesso studio, restringendo l'analisi solo alla popolazione affetta da DM1, il rischio di frattura femorale aumentava di 7 volte rispetto ai controlli. Sebbene, come si è già sottolineato, non tutti i dati in letteratura concordino, la sintesi delle evidenze induce ad affermare che il rischio di frattura è aumentato nella popolazione con diabete mellito, sia di tipo 1 sia di tipo 2, sebbene in questi ultimi la BMD risulti normale o aumentata rispetto alla popolazione normale [30]. Tale apparente paradosso potrebbe trovare una parziale spiegazione sia nella maggiore predisposizione a cadere [31] sia in una ridotta qualità ossea [32] che caratterizzerebbero il paziente con DM2.

\section{Alterazione della qualità del tessuto osseo nel diabete mellito}

L'acquisizione di un buon picco di massa ossea durante le prime decadi di vita è uno dei maggiori fattori protettivi nei confronti delle fratture.

Recentemente, uno studio prospettico della durata di un anno ha dimostrato che gli adolescenti con DM1 presentano una ridotta massa ossea e un minore volume osseo nonostante un normale sviluppo durante il periodo di crescita [33]. Infatti, una mineralizzazione scheletrica insufficiente durante la pubertà sarebbe il meccanismo determinante di una ridotta BMD nei soggetti adulti con DM1. La condizione osteopenica che compare nel DM1 suggerisce che i meccanismi demineralizzanti precedano i sintomi strettamente legati alla diagnosi di DM1; meccanismi autoimmunitari e antinfiammatori potrebbero svolgere un ruolo nella perdita di massa ossea e nell'alterata mineralizzazione ossea durante la pubertà, determinando un picco di massa ossea inferiore alla norma.

Nell'eziopatogenesi dell'osteoporosi nel paziente diabetico sembrano possedere un ruolo la microangiopatia [34] e la macroangiopatia [35] diabetica, in grado di indurre osteopenia.

La nefropatia diabetica si associa a una ridotta BMD in pazienti con DM1. È probabile che la progressiva riduzione della funzionalità renale induca un cambiamento del turnover osseo con prevalenza dell'azione osteoclastica fin dai primi stadi di insufficienza renale, capace di portare a una riduzione della BMD [36] sino ai quadri di marcata riduzione del turnover osseo propri della malattia adinamica dell'osso che caratterizza gli ultimi stadi della nefropatia cronica [37]. La stessa neuropatia, così come la presenza di vasculopatie periferiche sembrano rappresentare fattori determinanti o contribuenti alla ridotta massa ossea nei pazienti diabetici [38], anche se i meccanismi eziopatogenetici rimangono a oggi non chiariti.

Un nuovo capitolo di studio di grande rilevanza è rappresentato dal ruolo che possono esercitare fattori di crescita, interleuchine e glicazione del collagene nella fisiopatologia del tessuto osseo nel paziente diabetico. Così, negli ultimi anni sono stati pubblicati interessanti dati sul ruolo eziopatogenetico dell'Insulin-like Growth Factor (IGF-1) o dei composti avanzati di glicazione (AGE), oppure dei Peroxisome Prolifetor-Activate Receptors-gamma (PPAR-gamma), coinvolti nell'attività cellulare sia del tessuto adiposo sia del tessuto osseo (tabella 1).

L'IGF-1 è un peptide molecolarmente simile all'insulina: svolge un ruolo importante durante il periodo della crescita e continua ad avere un ruolo anabolico anche nella vita adulta. [39]. I livelli sierici di IGF-1 e di IGF-Binding-Protein 3 (IGFBP-3) sono ridotti nei pazienti con DM1 rispetto ai pazienti con DM2 oppure non diabetici. Inoltre, è stata dimostrata una correlazione positiva tra livelli sierici di IGF-1, IGFBP-3 e BMD [6]. 
Tabella 1 Fattori che influenzano il metabolismo osseo nel paziente diabetico.

\begin{tabular}{ll}
\hline Iperglicemia & $\begin{array}{l}\text { Ridotto riassorbimento osseo } \\
\text { Ridotto turnover osseo } \\
\text { Ridotta formazione ossea }\end{array}$ \\
\hline $\begin{array}{l}\text { Insulina e Insulin-like } \\
\text { Growth Factor }\end{array}$ & $\begin{array}{l}\text { Effetto anabolico sul tessuto } \\
\text { osseo } \\
\text { (IGF-1) }\end{array}$ \\
$\begin{array}{l}\text { Incremento dei livelli sierici } \\
\text { di estrogeni e androgeni }\end{array}$ \\
\hline $\begin{array}{l}\text { Accumulo di composti } \\
\text { avanzati di glicazione }\end{array}$ & Ridotto turnover osseo \\
(AGE) & \\
\hline
\end{tabular}

A conforto di questi dati Kemink et al. [40], studiando l'associazione tra BMD e parametri del turnover osseo, hanno osservato valori ridotti di BMD e dei livelli sierici di IGF-1, osteocalcina e fosfatasi alcalina nei pazienti con diabete mellito, a dimostrazione di una riduzione del turnover osseo in tale patologia.

Un aspetto altrettanto interessante della fisiopatologia del tessuto osseo nel paziente diabetico è il possibile effetto che l'accumulo degli AGE può esercitare sulle cellule ossee. Infatti, questi prodotti possono contribuire a peggiorare la resistenza del tessuto osseo accumulandosi nella componente collagenica della matrice ossea. Alcuni studi in vitro suggeriscono che l'accumulo di AGE stimolerebbe la produzione di interleuchina-6 da parte delle cellule ossee [41], inibirebbe l'espressione fenotipica degli osteoblasti [42] e, infine, incrementerebbe il riassorbimento osteoclastico [43]. A questo proposito, recentemente, Yamamoto et al. hanno studiato l'associazione tra i prodotti AGE, come le pentosidina, e l'incidenza di frattura vertebrale nella donna con DM2. La pentosidina è uno dei prodotti AGE più noto e facilmente misurabile. La sua concentrazione a livello dell'osso corticale trabecolare è inversamente associata alla resistenza meccanica dell'osso [44,45]. In questo studio [46] la pentosidina, ma non la BMD e il turnover osseo, risultava predittiva della frattura vertebrale nelle donne diabetiche, anche dopo correzione per i possibili fattori confondenti.

Come già ricordato, un ulteriore aspetto emergente della relazione tra osso e diabete è il possibile ruolo del PPARgamma nel metabolismo della cellula ossea. Infatti, gli osteoblasti e gli adipociti condividono un comune progenitore cellulare di tipo mesenchimale (Mesenchymal Stem Cells, MSC), che può maturare in cellula adiposa, osteoblastica o di altro tipo [47]. In questa via il recettore PPARgamma esercita un ruolo importante, infatti la sua maggiore espressione determina adipogenesi a discapito dell'osteoblastogenesi [48]. Al contrario, in condizione di totale mancanza di PPAR-gamma è stata dimostrato un incremento della massa ossea grazie a un'intensa osteoblastogenesi a partire da cellule progenitrici del midollo osseo [49]. Nei topi con DM1 è stata riscontrata una maggiore espressione del PPAR-gamma, inducendo a ipotizzare che il diabete insulino-dipendente contribuisca alla riduzione della massa ossea stimolando un accumulo di adipe al posto degli osteoblasti maturi. In maniera del tutto simile, è stato osservato che $i$ tiazolidinedioni (TZD), farmaci che attivano i recettori PPARgamma, stimolano la conversione delle cellule della linea osteoblastica in adipociti differenziati e contemporaneamente sopprimono in modo irreversibile l'espressione fenotipica degli osteoblasti [48-50].

\section{Fattori extrascheletrici}

L'apparente contraddizione di un rischio di frattura simile nei pazienti con DM1 o DM2, a fronte di una BMD normale o addirittura maggiore in questi ultimi, potrebbe essere semplicemente spiegata, oltre che dall'alterata qualità del tessuto osseo, anche dalla pesante influenza che fattori extrascheletrici possono esercitare nella patogenesi delle fratture nei pazienti diabetici (tabella 2 ). Infatti, la propensione a cadere causata da una ridotta acuità visiva, o da una cattiva propiocezione secondaria alla neuropatia diabetica oppure dalla nicturia che frequentemente affligge tali pazienti, costituisce un fattore amplificante il rischio di frattura nel soggetto con diabete mellito.

Alcuni studi, affrontando questo particolare aspetto, hanno dimostrato che nei pazienti diabetici il rischio relativo di frattura si riduce drasticamente, ma non si annulla, dopo correzione dei dati per i fattori predisponesti alla caduta, come la retinopatia, lo stroke, la neuropatia periferica e il deficit di forza muscolare [19]. In modo simile, Schwartz et al. [18] hanno riscontrato che le cadute, la ridotta acuità visiva, la ridotta attività fisica e l'utilizzo di benzodiazepine spiegano in piccola parte l'associazione tra diabete e frattura osteoporotica. Inoltre, due studi di tipo osservazionale molto importanti, come lo Health $A B C$ Study e il Women's Health Initiative Observational Study, hanno confermato una maggiore propensione alla caduta dei pazienti diabetici rispetto alla popolazione generale [51], in particolare in coloro con frattura rispetto a quelli senza frattura [38].

In tutti questi studi, comunque, la condizione diabetica è rimasta sempre associata alla frattura osteoporotica anche dopo correzione dell'analisi statistica per le complicanze diabetiche, suggerendo, pertanto, una relazione indipendente fra alterazioni del tessuto osseo e la patologia diabetica.

Si può quindi concludere che la ridotta qualità del tessuto osseo, indipendentemente dal fatto che la BMD sia normale o ridotta, contribuisca ad aumentare il rischio di frattura nel paziente diabetico, rischio già incrementato dalla maggiore propensione a cadere a causa delle comuni complicanze diabetiche quali un ridotta acuità visiva, la neuropatia periferica e il deficit di massa muscolare [52].

\section{Effetti sull'osso delle terapia ipoglicemizzante}

Esistono veramente pochi dati in letteratura riguardo all'effetto degli agenti ipoglicemizzanti sul tessuto osseo.

Tabella 2 Fattori di rischio di caduta nel paziente diabetico.

Ipoglicemia
Nicturia
Riduzione del visus
Ridotto equilibrio
Ipotensione ortostatica
Ridotta mobilità articolar

Nicturia

Ridotta mobilità articolare 
Circa l'interferenza dell'insulina, due importanti studi epidemiologici hanno riportato risultati contrastanti. Nel primo (studio caso-controllo) il trattamento con insulina non sembrava influenzare l'incidenza di frattura [53]; al contrario nel secondo studio, italiano, il trattamento insulinico ha determinato un significativo incremento della frattura ossea nell'uomo, mentre si confermava non significativo nella popolazione femminile [54]. Occorre tuttavia segnalare che nel subset di pazienti diabetici con una storia di malattia superiore a 3 anni, gli autori hanno riscontrato la mancanza di associazione fra trattamento insulinico e frattura ossea. Gli autori hanno suggerito che i pazienti in trattamento insulinico fossero anche quelli con una storia di malattia più lunga, con una maggiore incidenza di complicanze nonché i più soggetti a cadute, compresi quelli in giovane età, per le più frequenti crisi ipoglicemiche; tali condizioni avrebbero in parte nascosto il potenziale effetto positivo dell'insulina sul rischio di frattura, come sembrano dimostrare alcuni studi in vitro, riportando un effetto dell'insulina sulla proliferazione e la differenziazione della linea cellulare simil-osteoblastica nel ratto.

La metformina previene l'effetto negativo sulle cellule osteoblastiche associato all'accumulo degli AGE [55,56]. Questi dati di laboratorio sembrano confermare alcuni dati clinici che riportano una riduzione del $20 \%$ del rischio di frattura nei pazienti trattati con metformina [53].

Le sulfaniluree dispongono davvero di pochi dati relativi all'effetto diretto sul tessuto osseo. Alcuni studi epidemiologici suggeriscono, comunque, un effetto indiretto sull'incidenza di frattura ossea probabilmente spiegabile con il solo controllo glicemico [53].

Un nuovo e interessante capitolo di studio è rappresentato dall'effetto sul tessuto scheletrico dell'impiego di nuovi ipoglicemizzanti orali come i TZD. I TZD sono stati introdotti da circa 10 anni nel trattamento del DM2 e solo recentemente studi di una certa rilevanza hanno iniziato a evidenziare un loro possibile effetto negativo sul tessuto osseo.

Lo studio ADOPT (A Diabete Outcome Progression Trial) ha riportato un aumento del rischio di frattura fra le donne diabetiche in trattamento con rosiglitazone da almeno 4 anni quando venivano confrontate con una popolazione trattata con metformina o sulfaniluree [57]. Anche se una piccola parte di fratture riscontrate in questo studio riguardano siti ossei non tipici per fratture osteoporotiche, come tibia, mano e piede, il dato aggregato concorda con quelli riportati per altri TZD. Infatti, sia uno studio con rosiglitazone [58] sia un secondo studio con pioglitazone [59] hanno riferito un aumento del rischio di frattura nel sesso femminile ma non in quello maschile. Infine, un'analisi caso-controllo [60], ricavata da Meier et al. dal database della Medicina Generale del Regno Unito, ha confermato che i TZD aumentano il rischio di frattura nella donna con DM2 in postmenopausa, dimostrando un incremento di circa 3 volte del rischio per fratture del femore e per quelle non vertebrali.

Sebbene l'effetto negativo sul tessuto osseo dei TZD sembri tanto evidente, è corretto sottolineare che l'incidenza di frattura nei pazienti trattati con TZD è paragonabile a quella che caratterizza i pazienti con DM2 nel Women's Health Initiative Observational Study [51] e addirittura inferiore rispetto a quella riscontrata nei pazienti lungamente trattati con insulina [61]. Comunque, la terapia con TZD è associata a una riduzione sia della BMD misurata con DXA sia

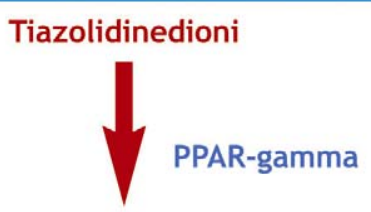

Cellula mesenchimale pluripotente del midollo osseo

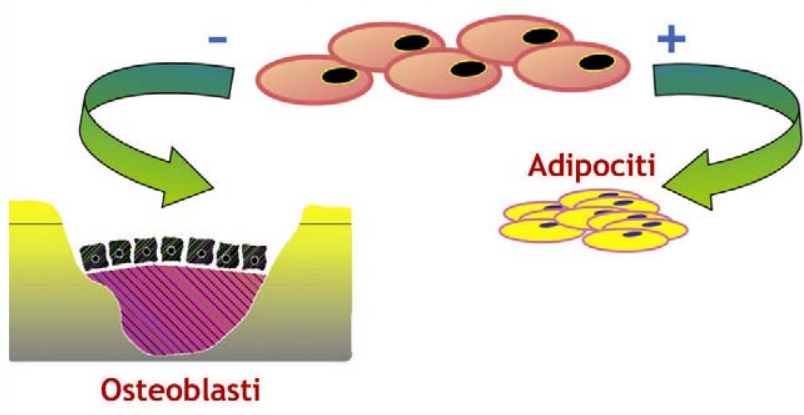

Fonte: modificata da Jiang Y, et al. Nature 2002;418(6893):41-9.

Figura 1 Effetto dei tiazolidinedioni sulle cellule mesenchimali pluripotenti.

dei livelli di formazione ossea determinati con i marker del turnover osseo [62].

Diverse ipotesi sono state avanzate per capire quali meccanismi possano spiegare il probabile effetto negativo dei TZD sul tessuto osseo. È stato dimostrato che i TZD sopprimono l'espressione dell'aromatasi e la sintesi degli estrogeni a livello del tessuto adiposo [63]; inibiscono, inoltre, la produzione ovarica di estradiolo e di testosterone, oppure sembrano in grado di stimolare la produzione di progesterone da parte del tessuto ovario [64].

Un ulteriore meccanismo possibile coinvolge l'attivazione del PPAR-gamma [65]. Infatti, questo recettore, che è espresso in numerosi tessuti compreso il tessuto osseo e adiposo, è in grado di orientare la differenziazione della cellula mesenchimale pluripotente verso la componente adiposa anziché verso quella osteoblastica [66] (fig. 1).

Una recente metanalisi della maggior parte degli studi condotti su pazienti con DM2 trattati con TZD ha confermato che questi farmaci hanno un effetto negativo sull'incidenza di frattura nella donna e non nell'uomo, e che tale effetto si amplifica con l'aumentare dell'età [67], suggerendo, pertanto, un risultato clinico dell'effetto diretto dei TZD sulle cellule ossee. Comunque, altri studi e approfondimenti sono necessari per affermare con ragionevole sicurezza che i TZD risultano dannosi per il tessuto osseo. È altresì vero che si dovrebbe essere perlomeno prudenti nella prescrizione di TZD in donne con osteoporosi, e in particolare nelle pazienti anziane.

\section{Prospettive future}

Negli ultimi anni sono state introdotte due nuove classi di farmaci nel trattamento del DM2: le incretine e gli inibitori dell'enzima dipeptidil-peptidasi-4 (DPP-IV).

Le incretine, il peptide glucagone-like (GLP-1) e il peptide insulinotropico glucosio-dipendente (GIP) sono sostanze 
ormonali secrete dalla mucosa intestinale in risposta al bolo alimentare, momento che sembra svolgere un ruolo rilevante nell'omeostasi del glucosio, modulando la secrezione di insulina glucosio-indotta.

In particolare, GLP-1 è un prodotto di trascrizione del gene del proglucagone ed è secreto principalmente dalle cellule $L$ intestinali. Una volta immesso in circolo questo peptide ha un'emivita inferiore a 2 minuti, dovuta alla rapida degradazione da parte dell'enzima DPP-IV. Oltre a tale meccanismo proprio del metabolismo glucidico, alcune evidenze dimostrano come questi peptidi ormonali secreti dalle cellule intestinali possano essere coinvolti nella regolazione in acuto e in cronico del metabolismo osseo. Infatti, le incretine sembrano modulare le interazioni che esisterebbero tra nutrizione e turnover osseo, come per esempio la soppressione postprandiale del riassorbimento osseo [68].

L'effetto che il GIP eserciterebbe sul tessuto osseo è stato ampiamente studiato in vitro e in vivo. Il recettore specifico per il GIP si ritrova espresso sulla superficie degli osteoclasti e incrementa sia l'espressione del collagene di tipo 1 sia l'attività della fosfatasi alcalina nelle cellule osteoblastolike. il GIP sembra altresì rallentare l'apoptosi osteoblastica con un effetto finale di tipo anabolico [69]; inibisce, inoltre, il riassorbimento indotto dall'ormone paratiroideo [70].

In vivo, topi knockout per il recettore del GIP mostrano un fenotipo di ridotta massa ossea secondario sia a una ridotta formazione ossea sia a un incremento del riassorbimento osseo [71]. Al contrario, in topi transgenici che sovraesprimono il GIP, la massa ossea risulta aumentata [72].

Per quanto riguarda il GLP-1, uno studio su topi knockout per i recettori del GLP-1 ha dimostrato un ruolo fondamentale di tali recettori nel controllo del riassorbimento osseo, apparentemente tramite una via calcitonino-dipendente. Questi topi presentano una riduzione dell'osso corticale e una fragilità ossea secondaria a un aumento del riassorbimento osseo, come documentano gli elevati livelli urinari dei cross-link [73].

Un altro studio recente, condotto con un'infusione continua di GLP-1 in ratti con DM2 e insulino-resistenza, ha dimostrato un effetto anabolizzante del GLP-1 sul tessuto osseo indipendentemente dai livelli dell'insulina [74].

Infine, l'exenatide, un peptide che possiede in parte una sequenza omologa al GLP-1, ma un'emivita ben più lunga del GLP-1, quando somministrato in modo continuo (per 3 giorni con pompa osmotica) determina effetti osteogenici in ratti con insulino-resistenza, e interagisce con la via Wnt promuovendo una formazione ossea e quindi un incremento della BMD [75].

A oggi non esistono dati in letteratura riguardo agli effetti sul metabolismo osseo e sulla BMD degli inibitori della DPP-IV, sitagliptin e vildagliptin. Comunque, considerando che l'effetto finale degli inibitori della DPP-IV è quello di prolungare l'azione del GLP-1, i loro effetti sul tessuto osseo potrebbero essere del tutto simili a quelli del GLP-1 e quindi di tipo osteoanabolizzante.

\section{Considerazioni conclusive}

In sintesi, i pazienti con DM1 risultano avere un rischio di frattura aumentato a causa della ridotta BMD e della maggiore propensione a cadere. La ridotta BMD è spiegata dal deficit di insulina e dall'iperglicemia, che influenza negativamente la formazione ossea. Inoltre, è noto come un prolungato e inadeguato controllo glicemico induca complicanze quali retinopatia, nefropatia e neuropatia diabetica, associate a una ridotta massa ossea; la retinopatia e la neuropatia incrementano entrambe il rischio di frattura predisponendo il paziente alla caduta.

D'altronde, i pazienti con DM2 presentano anch'essi un rischio di frattura aumentato a fronte di una BMD più elevata o normale. In questi pazienti, oltre alla propensione a cadere, i meccanismi che sottendono il maggior rischio di frattura sono un'alterata attività cellulare ossea e una scarsa qualità del tessuto osseo. Il turnover osseo è ridotto prevalentemente a causa dell'accumulo degli AGE, che sembrano in grado di influenzare le caratteristiche del collagene, oltre a influire negativamente sul reclutamento e l'attività osteoblastica. Pertanto, un controllo glicemico adeguato, un adeguato introito di calcio e vitamina $D$, uno screening per evidenziare una $B M D$ ridotta $e$, infine, la prevenzione e il trattamento delle complicanze diabetiche sono gli elementi chiave nella gestione dell'osteoporosi nei pazienti con entrambi i tipi di diabete. Particolare attenzione dovrebbe essere spesa per le donne diabetiche in trattamento con TZD, sopratutto se anziane.

Infine, è ragionevole affermare che nel caso dei pazienti diabetici con osteoporosi i farmaci che dovrebbero essere utilizzati sono gli stessi prescritti alla popolazione generale, pur riconoscendo che non esistono trial specifici per la popolazione diabetica.

\section{Conflitto di interesse}

Gli autori dichiarano di essere esenti da conflitto di interessi.

\section{Bibliografia}

[1] Johnell O, Kanis JA, Odén A, Sernbo I, Redlund-Johnell I, Petterson C, et al. Mortality after osteoporotic fractures. Osteoporos Int 2004;15(1):38-42.

[2] Jiang HX, Majumdar SR, Dick DA, Moreau M, Raso J, Otto DD, et al. Development and initial validation of a risk score for predicting in-hospital and 1-year mortality in patients with hip fractures. J Bone Miner Res 2005;20(3):494-500.

[3] Johnell O, Gullberg B, Kanis JA, Allander E, Elffors L, Dequeker $J$, et al. Risk factors for hip fracture in European women: the MEDOS Study. Mediterranean Osteoporosis Study. J Bone Miner Res 1995;10(11):1802-15.

[4] Janghorbani M, van Dam RM, Willett WC, Hu FB. Systematic review of type 1 and type 2 diabetes mellitus and risk of fracture. Am J Epidemiol 2007;166(5):495-505.

[5] Miazgowski T, Czekalski S. A 2-year follow-up study on bone mineral density and markers of bone turnover in patients with long-standing insulin-dependent diabetes mellitus. Osteoporos Int 1998;8(5):399-403.

[6] Jehle PM, Jehle DR, Mohan S, Böhm BO. Serum levels of insulinlike growth factor system components and relationship to bone metabolism in type 1 and type 2 diabetes mellitus patients. J Endocrinol 1998;159(2):297-306.

[7] Tuominen JT, Impivaara O, Puukka P, Rönnemaa T. Bone mineral density in patients with type 1 and type 2 diabetes. Diabetes Care 1999;22(7):1196-200. 
[8] Gallacher SJ, Fenner JA, Fisher BM, Quin JD, Fraser WD, Logue FC, et al. An evaluation of bone density and turnover in premenopausal women with type 1 diabetes mellitus. Diabet Med 1993;10(2):129-33.

[9] Weber G, Beccaria L, de' Angelis M, Mora S, Galli L, Cazzuffi MA, et al. Bone mass in young patients with type I diabetes. Bone Miner 1990;8(1):23-30.

[10] Alexopoulou O, Jamart J, Devogelaer JP, Brichard S, de Nayer P, Buysschaert $M$. Bone density and markers of bone remodeling in type 1 male diabetic patients. Diabetes Metab 2006;32(5 Pt 1): 453-8.

[11] Karagüzel G, Akçurin S, Ozdem S, Boz A, Bircan I. Bone mineral density and alterations of bone metabolism in children and adolescents with type 1 diabetes mellitus. J Pediatr Endocrinol Metab 2006;19(6):805-14.

[12] Mastrandrea LD, Wactawski-Wende J, Donahue RP, Hovey KM, Clark A, Quattrin T. Young women with type 1 diabetes have lower bone mineral density that persists over time. Diabetes Care 2008;31(9):1729-35.

[13] Cummings SR, Nevitt MC, Browner WS, Stone K, Fox KM, Ensrud KE, et al. Risk factors for hip fracture in white women. Study of Osteoporotic Fractures Research Group. N Engl J Med 1995; 332(12):767-73.

[14] Ivers RQ, Cumming RG, Mitchell P, Peduto AJ, Blue Mountains Eye Study. Diabetes and risk of fracture: The Blue Mountains Eye Study. Diabetes Care 2001;24(7):1198-203.

[15] Nicodemus KK, Folsom AR, lowa Women's Health Study. Type 1 and type 2 diabetes and incident hip fractures in postmenopausal women. Diabetes Care 2001;24(7):1192-7.

[16] Wakasugi M, Wakao R, Tawata M, Gan N, Koizumi K, Onaya T. Bone mineral density measured by dual energy $x$-ray absorptiometry in patients with non-insulin-dependent diabetes mellitus. Bone 1993;14(1):29-33.

[17] van Daele PL, Stolk RP, Burger H, Algra D, Grobbee DE, Hofman A, et al. Bone density in non-insulin-dependent diabetes mellitus. The Rotterdam Study. Ann Intern Med 1995;122(6):409-14.

[18] Schwartz AV, Sellmeyer DE, Ensrud KE, Cauley JA, Tabor HK, Schreiner PJ, et al., Study of Osteoporotic Features Research Group. Older women with diabetes have an increased risk of fracture: a prospective study. J Clin Endocrinol Metab 2001; 86(1):32-8.

[19] Forsén L, Meyer HE, Midthjell K, Edna TH. Diabetes mellitus and the incidence of hip fracture: results from the Nord-Trøndelag Health Survey. Diabetologia 1999;42(8):920-5.

[20] Barrett-Connor E, Holbrook TL. Sex differences in osteoporosis in older adults with non-insulin-dependent diabetes mellitus. JAMA 1992;268(23):3333-7.

[21] Isaia GC, Ardissone P, Di Stefano M, Ferrari D, Martina V, Porta M, et al. Bone metabolism in type 2 diabetes mellitus. Acta Diabetol 1999;36(1-2):35-8.

[22] Janghorbani M, Feskanich D, Willett WC, Hu F. Prospective study of diabetes and risk of hip fracture: the Nurses' Health Study. Diabetes Care 2006;29(7):1573-8.

[23] Korpelainen R, Korpelainen J, Heikkinen J, Väänänen K, Keinanen-Kiukaanniemi S. Lifestyle factors are associated with osteoporosis in lean women but not in normal and overweight women: a population-based cohort study of 1222 women. Osteoporos Int 2003;14(1):34-43.

[24] Kelsey JL, Browner WS, Seeley DG, Nevitt MC, Cummings SR. Risk factors for fractures of the distal forearm and proximal humerus. The Study of Osteoporotic Fractures Research Group. Am J Epidemiol 1992;135(5):477-89.

[25] Meyer HE, Tverdal A, Falch JA. Risk factors for hip fracture in middle-aged Norwegian women and men. Am J Epidemiol 1993;137(11):1203-11.

[26] Melton 3rd LJ, Achenbach SJ, O'Fallon WM, Khosla S. Secondary osteoporosis and the risk of distal forearm fractures in men and women. Bone 2002;31(1):119-25.
[27] Vogt MT, Cauley JA, Tomaino MM, Stone K, Williams JR, Herndon $\mathrm{JH}$. Distal radius fractures in older women: a 10-year follow-up study of descriptive characteristics and risk factors. The study of osteoporotic fractures. J Am Geriatr Soc 2002;50(1):97-103.

[28] Ahmed LA, Joakimsen RM, Berntsen GK, Fønnebø V, Schirmer H. Diabetes mellitus and the risk of non-vertebral fractures: the Tromsø study. Osteoporos Int 2006;17(4):495-500.

[29] Lipscombe LL, Jamal SA, Booth GL, Hawker GA. The risk of hip fractures in older individuals with diabetes: a population-based study. Diabetes Care 2007;30(4):835-41.

[30] Adami S. Bone health in diabetes: considerations for clinical management. Curr Med Res Opin 2009;25(5):1057-72.

[31] Schwartz AV, Hillier TA, Sellmeyer DE, Resnick HE, Gregg E, Ensrud KE, et al. Older women with diabetes have a higher risk of falls: a prospective study. Diabetes Care 2002; 25(10):1749-54

[32] Carnevale V, Romagnoli E, D'Erasmo E. Skeletal involvement in patients with diabetes mellitus. Diabetes Metab Res Rev 2004; 20(3):196-204.

[33] Moyer-Mileur LJ, Dixon SB, Quick JL, Askew EW, Murray MA. Bone mineral acquisition in adolescents with type 1 diabetes. J Pediatr 2004;145(5):662-9.

[34] Wientroub S, Eisenberg D, Tardiman R, Weissman SL, Salama R. Is diabetic osteoporosis due to microangiopathy? Lancet 1980;2(8201): 983.

[35] Vogt MT, Cauley JA, Kuller LH, Nevitt MC. Bone mineral density and blood flow to the lower extremities: the study of osteoporotic fractures. J Bone Miner Res 1997;12(2):283-9.

[36] Rigalleau V, Lasseur C, Raffaitin C, et al. Bone loss in diabetic patients with chronic kidney disease. Diabet Med 2007;24(1): 91-3.

[37] Pei Y, Hercz G, Greenwood C, Segre G, Manuel A, Saiphoo C, et al. Renal osteodystrophy in diabetic patients. Kidney Int 1993;44(1):159-64.

[38] Strotmeyer ES, Cauley JA, Schwartz AV, Nevitt MC, Resnick HE, Bauer DC, et al. Nontraumatic fracture risk with diabetes mellitus and impaired fasting glucose in older white and black adults: the health, aging, and body composition study. Arch Intern Med 2005;165(14):1612-7.

[39] Thrailkill KM, Liu L, Wahl EC, Bunn RC, Perrien DS, Cockrell GE, et al. Bone formation is impaired in a model of type 1 diabetes. Diabetes 2005;54(10):2875-81.

[40] Kemink SA, Hermus AR, Swinkels LM, Lutterman JA, Smals AG. Osteopenia in insulin-dependent diabetes mellitus; prevalence and aspects of pathophysiology. J Endocrinol Invest 2000; 23(5):295-303.

[41] Takagi M, Kasayama S, Yamamoto T, Motomura T, Hashimoto K, Yamamoto $\mathrm{H}$, et al. Advanced glycation endproducts stimulate interleukin- 6 production by human bone-derived cells. J Bone Miner Res 1997;12(3):439-46.

[42] Katayama Y, Akatsu T, Yamamoto M, Kugai N, Nagata N. Role of nonenzymatic glycosylation of type I collagen in diabetic osteopenia. J Bone Miner Res 1996;11(7):931-7.

[43] Miyata T, Notoya K, Yoshida K, Hone K, Maeda K, Kurokawa K, et al. Advanced glycation end products enhance osteoclastinduced bone resorption in cultured mouse unfractionated bone cells and in rats implanted subcutaneously with devitalized bone particles. J Am Soc Nephrol 1997;8(2):260-70.

[44] Hernandez CJ, Tang SY, Baumbach BM, Hwu PB, Sakkee AN, van der Ham F, et al. Trabecular microfracture and the influence of pyridinium and non-enzymatic glycation-mediated collagen cross-links. Bone 2005;37(6):825-32.

[45] Wang X, Shen X, Li X, Agrawal CM. Age-related changes in the collagen network and toughness of bone. Bone 2002;31(1):1-7.

[46] Yamamoto M, Yamaguchi T, Yamauchi M, Yano S, Sugimoto T. Serum pentosidine levels are positively associated with the presence of vertebral fractures in postmenopausal women with type 2 diabetes. J Clin Endocrinol Metab 2008;93(3):1013-9. 
[47] Karsenty G. Minireview: transcriptional control of osteoblast differentiation. Endocrinology 2001;142(7):2731-3.

[48] Lecka-Czernik B, Moerman EJ, Grant DF, Lehmann JM, Manolagas SC, Jilka RL. Divergent effects of selective peroxisome proliferator-activated receptor-gamma 2 ligands on adipocyte versus osteoblast differentiation. Endocrinology 2002;143(6):2376-84.

[49] Akune T, Ohba S, Kamekura S, Yamaguchi M, Chung UI, Kubota N, et al. PPARgamma insufficiency enhances osteogenesis through osteoblast formation from bone marrow progenitors. J Clin Invest 2004;113(6):846-55.

[50] Lecka-Czernik B, Gubrij I, Moerman EJ, Kajkenova O, Lipschitz DA, Manolagas SC, et al. Inhibition of Osf2/Cbfa1 expression and terminal osteoblast differentiation by PPARgamma2. J Cell Biochem 1999;74(3):357-71.

[51] Bonds DE, Larson JC, Schwartz AV, Strotmeyer ES, Robbins J, Rodriguez BL, et al. Risk of fracture in women with type 2 diabetes: the Women's Health Initiative Observational Study. $\mathrm{J}$ Clin Endocrinol Metab 2006;91(9):3404-10.

[52] Räkel A, Sheehy O, Rahme E, Le Lorier J. Osteoporosis among patients with type 1 and type 2 diabetes. Diabetes Metab 2008;34(3):193-205.

[53] Vestergaard P, Rejnmark L, Mosekilde L. Relative fracture risk in patients with diabetes mellitus, and the impact of insulin and oral antidiabetic medication on relative fracture risk. Diabetologia 2005;48(7):1292-9.

[54] Monami M, Cresci B, Colombini A, Pala L, Balzi D, Gori F, et al. Bone fractures and hypoglycemic treatment in type 2 diabetic patients: a case-control study. Diabetes Care 2008;31(2): 199-203.

[55] Cortizo AM, Sedlinsky C, MCCarthy AD, Blanco A, Schurman L. Osteogenic actions of the anti-diabetic drug metformin on osteoblasts in culture. Eur J Pharmacol 2006;536(1-2):38-46.

[56] Schurman L, MCCarthy AD, Sedlinsky C, Gangoiti MV, Arnol V, Bruzzone $L$, et al. Metformin reverts deleterious effects of advanced glycation end-products (AGEs) on osteoblastic cells. Exp Clin Endocrinol Diabetes 2008;116(6):333-40.

[57] Kahn SE, Haffner SM, Heise MA, Herman WH, Holman RR, Jones NP, et al., ADOPT Study Group. Glycemic durability of rosiglitazone, metformin, or glyburide monotherapy. N Engl J Med 2006;355(23):2427-43.

[58] Home PD, Jones NP, Pocock SJ, Beck-Nielsen H, Gomis R, Hanefeld $M$, et al., RECORD Study Group. Rosiglitazone RECORD study: glucose control outcomes at 18 months. Diabet Med 2007;24(6):626-34.

[59] Dormandy JA, Charbonnel B, Eckland DJ, Erdmann E, MassiBenedetti M, Moules IK, et al., PROactive investigators. Secondary prevention of macrovascular events in patients with type 2 diabetes in the PROactive Study (PROspective pioglitAzone Clinical Trial In macroVascular Events): a randomised controlled trial. Lancet 2005;366(9493):1279-89.

[60] Meier C, Kraenzlin ME, Bodmer M, Jick SS, Jick H, Meier CR. Use of thiazolidinediones and fracture risk. Arch Intern Med 2008;168(8):820-5.

[61] Schwartz AV, Sellmeyer DE, Vittinghoff E, Palermo L, LeckaCzernik B, Feingold KR, et al. Thiazolidinedione use and bone loss in older diabetic adults. J Clin Endocrinol Metab 2006;91(9): 3349-54.

[62] Grey A, Bolland M, Gamble G, Wattie D, Horne A, Davidson J, et al. The peroxisome proliferator-activated receptor-gamma agonist rosiglitazone decreases bone formation and bone mineral density in healthy postmenopausal women: a randomized, controlled trial. J Clin Endocrinol Metab 2007;92(4):1305-10.

[63] Rubin GL, Zhao Y, Kalus AM, Simpson ER. Peroxisome proliferator-activated receptor gamma ligands inhibit estrogen biosynthesis in human breast adipose tissue: possible implications for breast cancer therapy. Cancer Res 2000;60(6):1604-8.

[64] Seto-Young D, Paliou M, Schlosser J, Avtanski D, Park A, Patel P, Holcomb K, et al. Direct thiazolidinedione action in the human ovary: insulin-independent and insulin-sensitizing effects on steroidogenesis and insulin-like growth factor binding protein-1 production. J Clin Endocrinol Metab 2005;90(11): 6099-105.

[65] Hauner $\mathrm{H}$. The mode of action of thiazolidinediones. Diabetes Metab Res Rev 2002;18(Suppl 2):S10-5.

[66] Jiang $Y$, Jahagirdar BN, Reinhardt RL, Schwartz RE, Keene CD, Ortiz-Gonzalez XR, et al. Pluripotency of mesenchymal stem cells derived from adult marrow. Nature 2002;418(6893):41-9.

[67] Loke YK, Singh S, Furberg CD. Long-term use of thiazolidinediones and fractures in type 2 diabetes: a meta-analysis. CMAJ 2009;180(1):32-9.

[68] Clowes JA, Khosla S, Eastell R. Potential role of pancreatic and enteric hormones in regulating bone turnover. J Bone Miner Res 2005;20(9):1497-506.

[69] Bollag RJ, Zhong Q, Phillips P, Min L, Zhong L, Cameron R, et al. Osteoblast-derived cells express functional glucose-dependent insulinotropic peptide receptors. Endocrinology 2000;141(3): 1228-35.

[70] Zhong Q, Itokawa T, Sridhar S, Ding KH, Xie D, Kang B, et al. Effects of glucose-dependent insulinotropic peptide on osteoclast function. Am J Physiol Endocrinol Metab 2007;292(2): E543-8.

[71] Xie D, Cheng H, Hamrick M, Zhong Q, Ding KH, Correa D, et al. Glucose-dependent insulinotropic polypeptide receptor knockout mice have altered bone turnover. Bone 2005;37(6): 759-69.

[72] Xie D, Zhong Q, Ding KH, Cheng H, Williams S, Correa D, et al. Glucose-dependent insulinotropic peptide-overexpressing transgenic mice have increased bone mass. Bone 2007;40(5): 1352-60.

[73] Yamada C, Yamada Y, Tsukiyama K, Yamada K, Udagawa N, Takahashi N, et al. The murine glucagon-like peptide-1 receptor is essential for control of bone resorption. Endocrinology 2008;149(2):574-9.

[74] Nuche-Berenguer B, Moreno P, Esbrit P, Dapía S, Caeiro JR, Cancelas J, et al. Effect of GLP-1 treatment on bone turnover in normal, type 2 diabetic, and insulin-resistant states. Calcif Tissue Int 2009;84(6):453-61.

[75] Nuche-Berenguer B, Moreno P, Portal-Nuñez S, Dapía S, Esbrit P, Villanueva-Peñacarrillo ML. Exendin-4 exerts osteogenic actions in insulin-resistant and type 2 diabetic states. Regul Pept 2010;159(1-3):61-6. 